\title{
FPGA Realization of Power Quality Disturbance Detection: An Approach with Wavelet, ANN and Fuzzy Logic
}

\begin{abstract}
Identification and classification of voltage and current disturbances in power systems is an important task in power system monitoring and protection. Most power quality disturbances are non-stationary and transitory and the detection and classification have proved to be very demanding. New intelligent system technologies using wavelet transform, expert systems and artificial neural networks provide some unique advantages regarding fault analysis. This paper presents new approach aimed at automating the analysis of power quality disturbances including sag, swell, transient, fluctuation, interruption and normal waveform. The approach focuses on the application of discrete wavelet transform technique to extract features from disturbance waveforms and their classification using a powerful combination of neural network and fuzzy logic. The system is modelled using VHDL followed by extensive testing and simulation to verify the correct functionality of the system. Then, the design is synthesized to Mercury EPIM120F484C5 FPGA, tested and validated. Comparisons, verification and analysis made from the results obtained from the application of this system on software-generated and utility sampled disturbance signals validate the utility of this approach and achieved a classification accuracy of $98.17 \%$.
\end{abstract}

Key-Words: - Power quality, neural network, fuzzy logic, discrete wavelet transform, VHDL, FPGA, classification

\section{Introduction}

Power Quality (PQ) has become a major concern to both electric utilities and customers. There are endless numbers of system configurations, making each $\mathrm{PQ}$ problem unique in its characteristics and diagnostic. The growing concern about $P Q$ in this decade is based largely on the daily increased use of modern sensitive computing equipment and other non-linear loads [1]. In addition, industries requiring very high availability of service and precision manufacturing systems are very sensitive to PQ problems.

The disturbance waveforms contain serious imprecision of data and provides very little information on $\mathrm{PQ}$, making conventional programs fail to identify $\mathrm{PQ}$ problems. Thus, with the lack of $P Q$ knowledge resources, the explanation facility of an expert system becomes an invaluable asset.

The advantages of an automated system for fault analysis have been recognized in the late 60 -ties. Over the last ten years a number of different approaches to the automated fault analysis using intelligent methods were suggested. There are few works that had been done in this area. In one of the work [2], proposes a classification scheme using a neural network trained to recognize patterns of transmission line faults is incorporated in a PC-based system that analyses data files from substation digital fault recorders. This system analyses data files coming from the digital fault recorders located in substations. The role of the neural net in this solution is to process current signals and identify the one with the largest disturbance. Furthermore, it will try to classify disturbance according to the fault type. Another work [3] utilizes a rule-based method and a wavelet packetbased hidden Markov model (HMM) for the classification of power disturbances. The rule-based method classifies timecharacterized-feature disturbances and the wavelet packetbased HMM is utilized for the frequency-characterized feature power disturbances. Lastly, a classifier to carry out waveform recognition in the wavelet domain using multiple neural networks is proposed by Surya et al. [4]. The classifier provides a degree of belief for the identified waveform. The degree of belief gives an indication about the goodness of the decision made. The wavelet-based classifier is equipped with an "acceptance threshold", which is an agreement level threshold (for the voting scheme), to accept a decision made for the identified waveform.

Wavelet transforms have been proven to be very efficient in signal analysis [5]. The use of wavelets for PQ studies is most appropriate because most disturbances on a power supply system are non-stationary transients such as those due to capacitor switching and other switching impulses. The wavelet transform represents time-dependent signal behaviour in both the time and scale (frequency) domains or the wavelet domain.

Artificial neural network (ANN) has attracted a great deal 
of attention because of their pattern recognition capabilities, and their ability to handle noisy data. On the other hand, fuzzy logic (FL) is an indispensable tool for PQ analysis. The value of FL arises from the imprecision and vagueness attached to PQ data measurements. Fuzzy logic and neural networks are natural complementary tools in building intelligent systems. While ANNs are low-level computational structures that perform well when dealing with raw data, FL deals with reasoning on a higher level, using linguistic information. However, fuzzy systems lack the ability to learn and cannot adjust themselves to a new environment. On the other hand, although neural networks can learn, they are opaque to the user. Integrating ANN and FL can combine the parallel computation and learning abilities of neural networks with the human-like knowledge representation and explanation abilities of fuzzy systems. As a result, neural networks become more transparent while fuzzy systems become capable of learning. Therefore, a combination of both methods is able to solve the limitations stated above.

However, the classification methods employed in the works $[2,3,4]$ mentioned above are based mainly on software implementation. Classifiers developed in software have several drawbacks. A limiting factor is that the size of classifier is limited by the size of the system that runs the code for the classifier system. Field Programmable Gate Array (FPGA) implementations remain essential for taking full advantage of parallelism and provide an increase in speed and performance [6]. This allows all of the system calculations to be done directly in parallel. This contributes a significant increase in performance compared to a systems implemented in software where the many internal calculations compiled into multiple individual commands for a processor to complete sequentially.

Huang et al. [7] proposed a classifier utilizing a combination of all three technologies developed in software. In this paper, the authors present a similar classifier but with a different methodology combining the three individual technologies; wavelet transform, neural network and fuzzy logic realizing on FPGA resulting in a simpler classifier with a significant increase in speed, performance and accuracy.

This work also presents a new algorithm incorporating artificial intelligence using VHDL that is fast, simple and easy to debug or make changes. These added benefits coupled with a short design cycle definitely contribute to the area of $P Q$ disturbance classifications.

\section{Design Overview}

Detection of PQ disturbances consists of two steps: feature extraction and classification. In this work, the detection of PQ disturbance is performed based on the model shown in Figure 1.

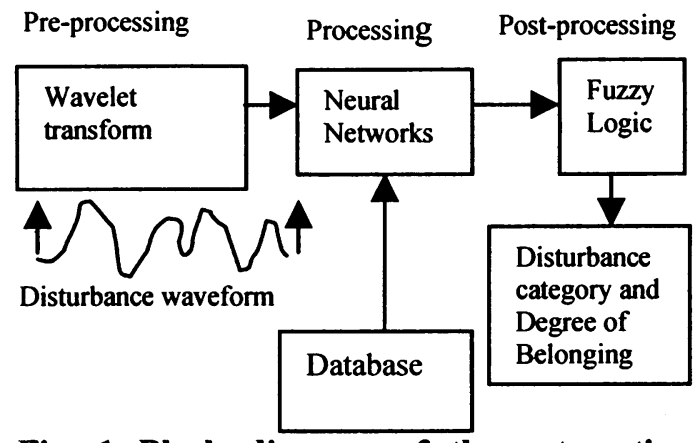

Fig. 1 Block diagram of the automatic disturbance recognition system

In the waveform generation, six types of disturbances include transients, sag, swell, interruption, fluctuation and perfect wave are randomly superimposed on the normal waveform at a rate in accordance with their relative occurring frequencies. The definitions and descriptions of these classes are as those given by PQ Network [8]. In this work, the power frequency chosen is $60 \mathrm{~Hz}$. The disturbance waveform consists of five cycles of samples of three-phase voltage signals (in per unit).

The samples are fed in as inputs to the pre-processing stage. The pre-processing stage is used to perform feature extraction to extract the disturbance information from the PQ disturbance signal. The disturbance signals are sampled and pre-processed by a 5-level discrete wavelet transform for feature extraction. Since the sampling frequency is set as $256 f_{1}$ with $f_{1}$ as the power frequency, the transform coefficients contain the information about the original waveform as shown in Table 1 .

Important information is extracted from the distorted waveform based on the features in the decomposed waveform. Wavelet decomposition is achieved by a combination of high and low pass filters. The obtained coefficients are very useful, as the large coefficients determine where there are steep gradients or discontinuities in the function. Consider the DWT for a signal $f(t) \in L^{2}(R)$ as stated in equation 1 .

$\mathrm{CA}_{0}{ }^{(\mathrm{n})} \mathrm{f}=\mathrm{f}(\mathrm{n})$

where $f(n)$ is the approximation of $f(t)$ at scale- 0$)$. The $f(n)$ signal can then be expressed with a multiresolution representation as stated in equation 2 .

$\left\{C A_{\mathrm{J}},\left\{\mathrm{CD}_{\mathrm{j}}\right\}_{1 \leq j \leq \mathrm{j}}\right\}$

should a level J-level DWT decomposition be adopted, where $C A_{J}=\left\{C A_{J}^{(n)} f\right\}$ and $C D_{j}=\left\{C D_{j}^{(n)} f\right\}, 1 \leq j \leq J$. The output of the discrete wavelet transform scheme is the feature vectors, i.e., transformed data comprising the approximation coefficients and the detailed coefficients denoted by $C_{5}$ and $C D_{1} \sim \mathrm{CD}_{5}$ respectively representing a particular disturbance signal. These coefficients, instead of the original waveforms or the whole transformed data is fed in as pattern inputs to the processing stage to perform pattern recognition and provide an output associated with that pattern. 
Table 1 Sub-bands of WT transform coefficients

\begin{tabular}{|l|l|}
\hline Transform coefficients & Frequency range \\
\hline$C D_{1}$ & $64 \mathrm{f}_{1} \sim 128 \mathrm{f}_{1}$ \\
\hline $\mathrm{CD}_{2}$ & $32 \mathrm{f}_{1} \sim 64 \mathrm{f}_{1}$ \\
\hline $\mathrm{CD}_{3}$ & $16 \mathrm{f}_{1} \sim 32 \mathrm{f}_{1}$ \\
\hline $\mathrm{CD}_{4}$ & $8 \mathrm{f}_{1} \sim 16 \mathrm{f}_{1}$ \\
\hline $\mathrm{CD}_{5}$ & $4 \mathrm{f}_{1} \sim 8 \mathrm{f}_{1}$ \\
\hline $\mathrm{CA}_{5}$ & $0 \sim 4 \mathrm{f}_{1}$ \\
\hline
\end{tabular}

In this work, a combination of ANN and FL is proposed for disturbance classification. The processing stage is made of an ANN block trained to recognize patterns of input/output pair from the disturbance database. The ANN is trained before it is utilized in the classification process. The processing stage operates in two different modes: Training and Evaluation as shown in Figure 2.

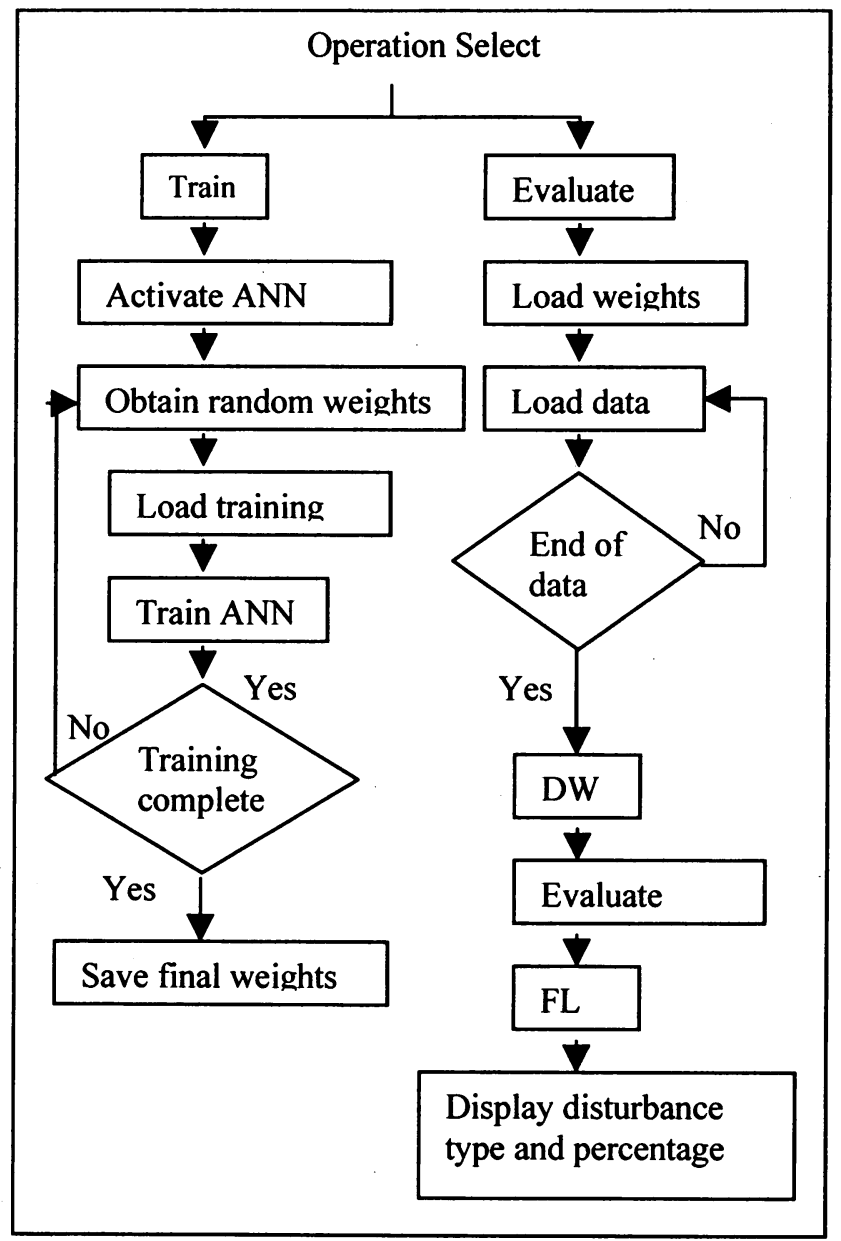

Fig. 2 Modes of operation

The purpose of training is to obtain the most appropriate weight values based on training data and to enable the ANN to learn from the patterns provided. When training mode is selected, DWT and FL blocks are disabled (inactive) and only the ANN block is activated. This system trains the onchip ANN by passing training data taken from the data input port in the following format in Figure 3.

\begin{tabular}{|l|l|l|}
\hline Target & Input2 & Input1 \\
\hline
\end{tabular}

Fig. 3 Training data format

The system trains the network using the provided data for a user-specified number of iterations; in this project 2000 iterations were used. When the ANN has been trained, the final weights are sent to the output port and the training of the ANN is complete. The final weight values were assigned to each neuron when performing classification.

In Evaluation (testing) mode, the DWT block accepts and processes inputs from the input port consisting of $P Q$ disturbance data. When there is no more input, the block processes and outputs the values of approximate and detail coefficients respectively to the ANN block. The ANN block operates based on command type received from the Data Provider block. The ANN block processes the coefficients and sends the evaluated output results to the FL block for classifying the type of disturbance together with the percentage of belonging to a particular disturbance class. The system functions in five different scenarios. The 3-bit value on the select input port determines the mode of operation. There are five possible scenarios:

$\begin{array}{ll}1 . & 001 ": \text { Train } \\ 2 . & 010 ": \text { Train from RAM } \\ 3 . & 011 ": \text { Send weights home } \\ 4 . & 100 ": \text { Load weights } \\ 5 . & \text { "101": Evaluate neural network }\end{array}$

The algorithm chosen for the ANN implementation was the Univariate randomly optimized Neural Network (uronn) [9]. This algorithm is robust for comparing training speeds between software and hardware. This algorithm is very advantageous approach in implementing ANN because less calculation needs to be performed in each epoch as this algorithm searches for the weights that best fit the network by randomly changing them in order to minimize error.

Finally, the post-processing stage groups the output data and form decisions. In order to obtain an accurate result, the output results are then fed into the FL block to further classify the output in one of the six disturbance types based on a set of predefined codeword. The rule-based postprocessing improves the accuracy of the classification result.

The system is designed to recognize six types of $P Q$ disturbances. These are identified by taking readings of the voltage values (in per unit) of each cycle in the time domain. The values are then compared against the preset characteristic of a particular PQ disturbance as defined by the IEEE 1159.2 working group. The ANN is trained with samples of disturbance data from each class. Fuzzy rules were established to perform the classification task. 


\section{Description of VHDL Modules}

The system construction of the PQ disturbance classifier is based on the block diagram as shown in Figure 4.

\subsection{Discrete Wavelet Transform (DWT)}

The $f(n)$ signal is passed through a low-pass digital filter $(h d(n))$ and a high-pass digital filter $(g d(n))$. Wavelet coefficients of approximation and of detail respectively are determined using equation 3 and 4 [10]:

$\mathrm{cA}_{\mathrm{i}}(\mathrm{n})=\Sigma_{\mathrm{k}} \mathrm{f}(\mathrm{n}) * \mathrm{~h}_{\mathrm{d}}(-\mathrm{k}+2 \mathrm{n})$

$\mathrm{cD}_{\mathrm{i}}(\mathrm{n})=\Sigma_{\mathrm{k}} \mathrm{f}(\mathrm{n}) * \mathrm{~g}_{\mathrm{d}}(-\mathrm{k}+2 \mathrm{n})$

The process of coefficients determination iterates and this procedure provides the approximated $(a j(n))$ and the detailed $(\operatorname{dj}(n))$ version of the original signal as well as the corresponding wavelet spectrum [11].

\subsection{Artificial Neural Network}

The ANN block is made of Neural Network and Control Unit block.

- Neural Network: A neural network consists of simple processing elements known as neurons. The neurons perform a linear combination of input vector $\mathrm{x}$ with the weight vector $m_{k}$, as shown in equation 5 .

$\mathrm{u}_{\mathrm{k}}=\mathrm{mx}^{\mathrm{T}}=\Sigma \mathrm{m}(\mathrm{k}, \mathrm{i}) \mathrm{x}(\mathrm{i})$ for $\mathrm{i}$ from 1 to $\mathrm{P}$

- Control Unit: The control unit is used to control the neural network. It consists of five sub modules modelled in a top-down design approach [12] as described below.

Trainer: This module basically utilizes state machines governing control signals for the neural network.

Bus Master: This component is designed to get and set the weights and to activate the neural network during operational mode. It consists of a data bus, address bus, command bus and select bus.

Error calculator: Error calculator instructs the control unit to keep or discard a new weight.

Pseudo random number generator: It generates the random weights employing linear feedback shift register (LFSR) using equation 6.

$\sum_{i=0}^{n} c_{i} s_{i+k}=0, \mathrm{k} \geq 0$

where $s$ is the input sequence of length $n$ and $c_{n}=1$ by definition [13].

RAM interface: This module handles the storage of the training data into RAM. It also recalls the training data set for the control unit for subsequent training epochs.

\subsection{Fuzzy Logic}

A fuzzy classifier consists of three parts, the fuzzification of the inputs, the defuzzification of the outputs and the rulebase.

\section{- Implementation of fuzzifier}

The function of fuzzifier is to transform crisp inputs into fuzzy inputs. To get fuzzy inputs, the crisp input is compared with membership function parameters.

- Implementation of Rule Evaluation

Rule evaluation determines what control action should occur in response to a given set of input values. The rule evaluation method used in this design is 'min-max' inferences [14].

- Implementation of defuzzifier

Defuzzification transforms fuzzy output to crisp output based on output membership function. Centre of Gravity (COG) or centroid method [14] as shown in equation 7 is used to determine the crisp output.

Output $=(\Sigma$ fuzzy output * singleton $) /(\Sigma$ fuzzy output $)$

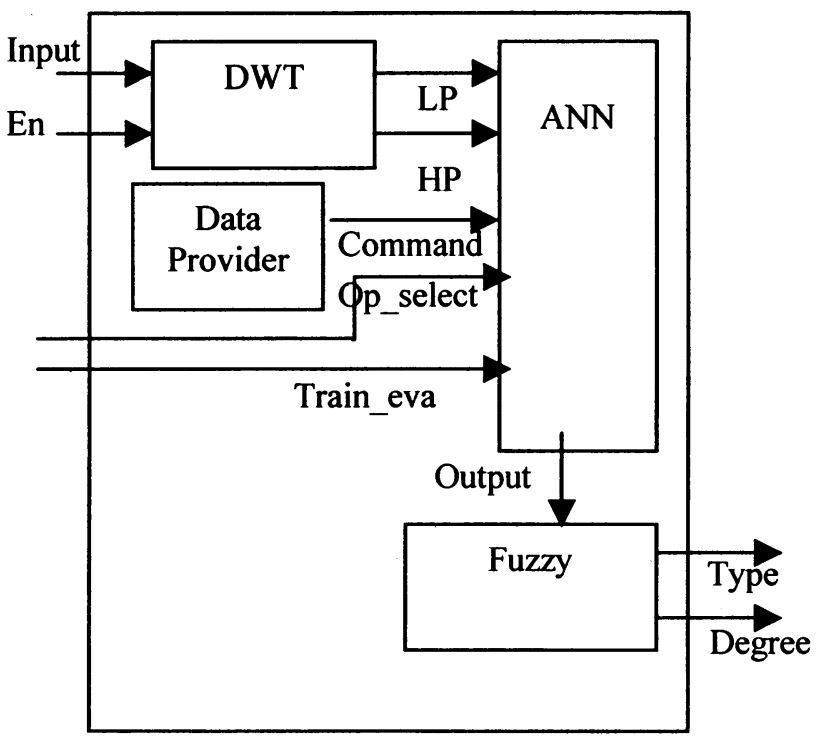

Fig. 4 High-level system design

\subsection{Data Provider}

This module accepts and processes inputs from the ANN and provides appropriate input for the system to operate.

\section{Results and Discussion}

\subsection{Data Collection}

Simulation of $P Q$ disturbances is performed by thirdparty tools Alternative Transient Program (ATP), Matlab and COMTRADE). A total of 10200 examples (1700 examples per class) with different starting time, duration and distortion magnitude were used to test the classification method. Collaboration was made with Tenaga Research Centre's PQ Lab. Field data was collected from six different substations from throughout Malaysia include Bayan Lepas, Bukit Raja, Serdang, Skudai, Bangsar and TNB HQ.

\subsection{VHDL Simulation and Results}

The results of classification using test and training set for 
all six disturbances are shown in Table 2. The table is organized as follows: in each row the performance of the network is represented by a specific PQ disturbance type, each column represents the results of the classification. The percentage of correct classification is calculated using equation 8 .

$\%$ correct classification rate $=$ Total correct classified disturbance events / total disturbance events

Table 2 Neural network performances on the test data (C-class, 1-transient, 2-voltage sag, 3-voltage swell, 4interruption, 5-fluctuation, 6-normal, Correctpercentage of correct identification, mi-percentage of mistake identification to class i)

\begin{tabular}{|l|l|l|l|l|l|l|l|}
\hline C & Correct & M1 & M2 & M3 & M4 & M5 & M6 \\
\hline 1 & 97.24 & - & 0.6 & 0.8 & - & 1.1 & 0.26 \\
\hline 2 & 98.87 & 0.4 & - & - & - & 0.73 & - \\
\hline 3 & 97.14 & 1.15 & - & - & 0.1 & 1.26 & 0.35 \\
\hline 4 & 98.20 & 0.03 & - & - & - & 1.54 & 0.23 \\
\hline 5 & 97.73 & 1.27 & 0.2 & 0.8 & - & - & - \\
\hline 6 & 99.98 & - & 0.01 & 0.01 & - & - & - \\
\hline
\end{tabular}

In Figure 5, the DWT block accepts 8-bit value of PQ disturbance data and processes the data to extract important features. At the falling edge of ENA (which indicates the end of sample data), the values of detail and smooth coefficients of the sample data is sent to the output coupled with an active Ready signal to indicate the values available at the output.

Figure 7 shows the final weights for all six neurons at the end of the ANN training process. The ANN is trained using a large number of training samples before they are used as part of the classifier. Thus, an input vector file containing all the training values is created. During a training run, a random weight is generated for one weight in the neural net, and the output is evaluated. If this new weight improves the performance of the system it replaces the old weight; otherwise the original weight is kept. The training algorithm used performs the calculation through error calculator for each input set six times per neuron, resulting in a final best weight for the neurons.

In Figure 7, the fuzzy logic block accepts crisp inputs from the ANN and performs the final classification into the appropriate disturbance type. In addition, it also provides the degree of belonging of a particular disturbance to the disturbance class. This proves to be very useful, as it is a measure of the severity and critical level of a power disturbance event.

In order to classify the six disturbances, individual codeword is predefined and assigned to each class. An output of " 001 " on the result register represents a transient disturbance, " 010 " represents a sag, " 011 " represents a normal waveform, "100" represents a swell, "101" represents an interruption and "110" represents fluctuation. Based on the results obtained, the system worked as expected and successfully classified all six disturbances.

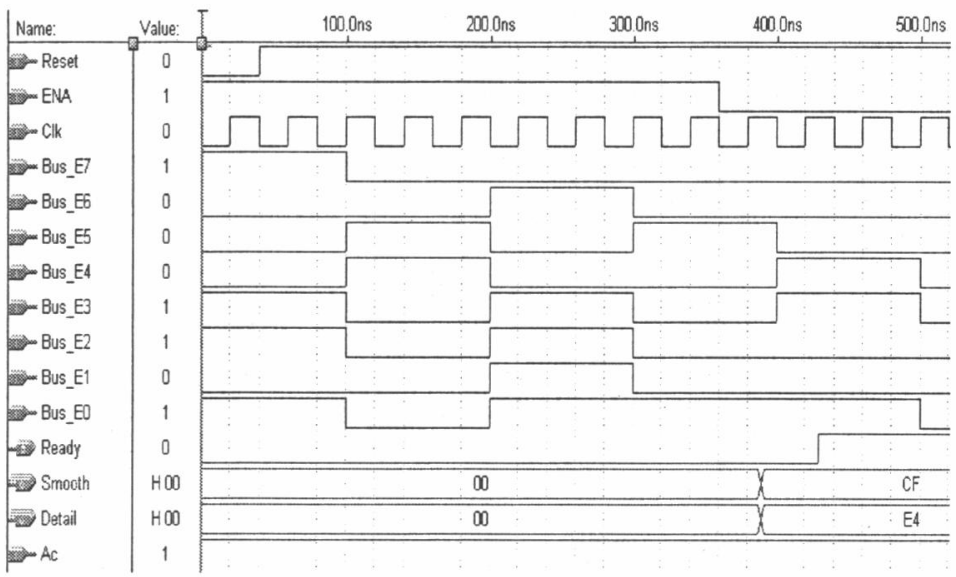

Fig. 5 Discrete wavelet transform output

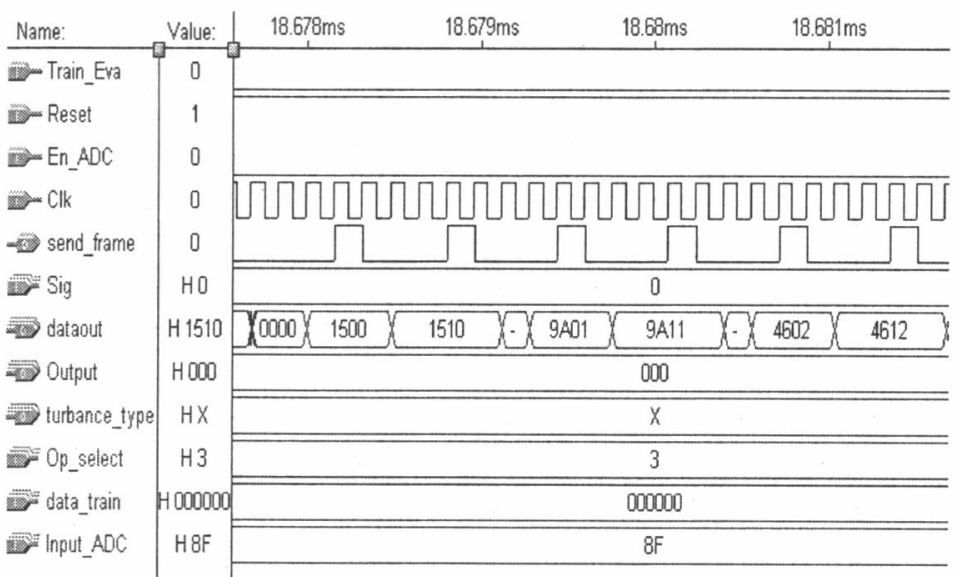

Fig. 6 ANN output (training stage)

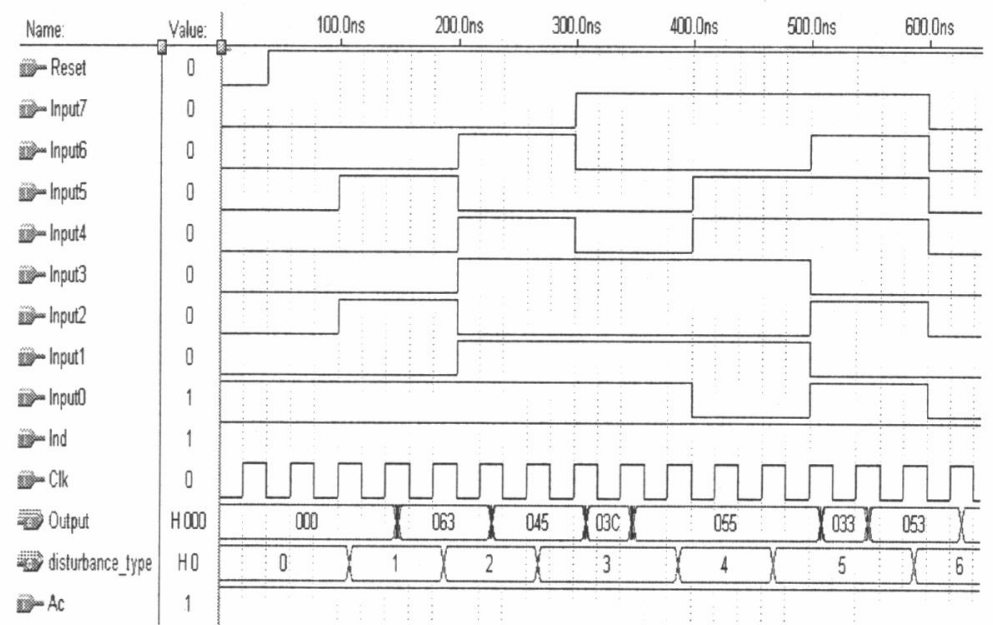

Fig. 7 Fuzzy logic output

\subsection{Synthesis Results}

In optimising the design, a balance between frequency and area was taken into account to select a suitable device. The project was successfully synthesized using Mercury 
EP1M120F484C5 device family from Altera Corp. Table 3 shows a sample of the synthesis report generated by Quartus II version 2.2 after successfully synthesizing the project.

Table 3 Synthesis report

\begin{tabular}{|l|l|}
\hline Processing status & Fitting successful \\
\hline Timing requirements & Requirements met \\
\hline Chip name & Project \\
\hline Device for compilation & EP1M120F484C5 \\
\hline Percentage of LC used (\%) & $1,432 / 4,800(29 \%)$ \\
\hline Maximum Frequency (MHz) & 121.95 \\
\hline Registers & $658 / 5,733(11 \%)$ \\
\hline I/O Pins & $69 / 311(22 \%)$ \\
\hline
\end{tabular}

\subsection{Comparison}

In order to examine and analyse the effectiveness and feasibility of the proposed approach, a comparison in terms of percentage of accuracy is made and presented in Table 4, which shows that a combination of more than one method yields a better result.

\section{Table 4 Performance comparisons}

\begin{tabular}{|l|l|}
\hline Method & Accuracy \\
\hline Wavelet Transform and Neural Fuzzy [7] & 96.5 \\
\hline Wavelet-based Neural Classifier [4] & 94.37 \\
\hline Proposed & 98.17 \\
\hline Neural Network [2] & 95.93 \\
\hline Wavelet and Fuzzy Logic [3] & 98.7 \\
\hline
\end{tabular}

Method by Jaehak et al. [3] yielded the highest result. However, the response time of a fuzzy based classifier for classifying disturbance signals is rather slow. In addition, as the number of rules grows, the system response time slows down. In comparison, this work yields a shorter classification time of 16 microseconds. This shows that limitations exist for software implementation in which they will never be fast enough compared to hardware platforms.

In one of the work [15], the wavelet transform circuit is made through two FPGA chips, which are XC4010XL IC developed by Xilinx, Inc. The maximum frequency achieved is $80 \mathrm{MHz}$. The total number of logic cells utilized in the two FPGA chips is 8184 and 8160 respectively. In comparison, this method utilized only 1432 logic cells and could run at a frequency of up to $121.95 \mathrm{MHz}$. The proposed method exhibits a good balance between area and frequency, which is vital in any hardware design.

\section{Conclusions and Future Work}

A new approach of hardware prototyping for the realization of PQ disturbance classifier utilizing wavelet transform, neural network and fuzzy logic is proposed. The initial work involved determining the functions of the system and designing the individual modules in VHDL. The functionality and timing behaviour of the modules were successfully verified. Finally, the system was synthesized to the Mercury EP1M120F484C5 FPGA device. The performance of the classifier is encouraging. The results reveal that the proposed approach is computationally simple, accurate and exhibits a good balance of flexibility, speed, size and design cycle time. These important characteristics are needed to design a good classification system. Comparison and results presented validate the successful classification of $\mathrm{PQ}$ disturbance.

\section{References}

[1] Math H.J. Bollen, "Understanding Power Quality Problems: Voltage Sags and Interruptions", IEEE, Inc., New York, pp. 139$253,2000$.

[2] Mladen Kezunovi, Igor Rikalo, "Detect and Classify Faults Using Neural Nets", IEEE Computer Applications in Power, ISSN 0895-0156, pp. 42-47, October 1996.

[3] Jaehak Chung, Edward J. Powers, W. Mack Grady, Siddharth C. Bhatt, "Power Disturbance Classifier Using a Rule-Based Method and Wavelet Packet-Based Hidden Markov Model", IEEE Transactions on Power Delivery, Vol. 17, No. 1, pp. 233-241, January 2002.

[4] Surya Santoso, Edward J. Powers, W. Mack Grady, Antony C. Parsons, "Power Quality Disturbance Waveform Recognition Using Wavelet-Based Neural Classifier - Part 2: Application", IEEE Transactions on Power Delivery, Vol. 15, No. 1, pp. 229235, January 2000.

[5] A.M. Gaouda, M.M.A. Salama, M.R. Sultan, A.Y. Chikhani, "Power Quality Detection and Classification Using WaveletMultiresolution Signal Decomposition", IEEE Transactions on Power Delivery, Vol. 14, No. 4, pp.1469-1476, October 1999.

[6] F. Mohd-Yasin, M.B. Ibne-Reaz, M.S. Sulaiman, F. Choong, A. Alaudeen, "Design of Digital Residential Energy Meter Employing VHDL", Proceedings of the IEEE International Conference on Consumer Electronics, Sydney, Australia, 4 pages, December 2003.

[7] Jiansheng Huang, Michael Negnevitsky, D. Thong Nguyen, "A Neural-Fuzzy Classifier for Recognition of Power Quality Disturbances", IEEE Transactions on Power Delivery, Vol. 17, No. 2, pp. 609-616, April 2002.

[8] PQ Network, "PQ Definitions", World Wide Web URL at http://www.mtm.at/pqnet/PQDEF.htm, March 2004.

[9] Looney, Carl, Pattern Recognition Using Neural Networks: Theory and Algorithms for Engineers and Scientists, New York: Oxford University press, 1997, Chapters 3, 4 and 6.

[10] Robertson, D.C., Camps, O. I., Mayer, J.S., "Wavelets and electromagnetic power system transients", IEEE Transactions on Power Delivery, Vol. 11, No. 2, pp.924-930, April 1996.

[11] Penna, C., "Detection and classification of power quality disturbances using the wavelet transform", M. Sc .Dissertation, Universidade Federal de Uberlandia, Brazil, June 2000.

[12] Stefan Sjoholm, Lennart Lindh, VHDL for Designers, Prentice Hall, 1997, pp. 261-263.

[13] "Linear Feedback Shift Registers", World Wide World Wide Web URL at

http://www.math.cudenver.edu/ wcherowi/courses/m5410/m5410f sr.html, pp 1-8, 09 January 2002.

[14] K. Asai, "Fuzzy Systems for Information Processing", Ohmsha, Ltd. Tokyo, Japan, 1995.

[15] Shyh-Jier Huang, Tsai-Ming Yang, Jiann-Tseng Huang, "FPGA Realization of Wavelet Transform for Detection of Electric Power System Disturbances", IEEE Transactions on Power Delivery, Vol. 17, No. 2, pp. 388-394, April 2002. 